\title{
ANALOG CIRCUITS DESIGN AUTOMATION TOOL USING GENETIC ALGORITHMS
}

\author{
Y. M. A. Khalifa \\ Electrical Engineering Department, University of North Dakota, Grand Forks, ND 58202, USA \\ D. H. Horrocks \\ School of Engineering, Cardiff University of Wales, Cardiff CF2 3TF, UK
}

\begin{abstract}
An investigation into the application of Genetic Algorithms (GA) for the design of electronic analog circuits is presented in this paper. In this paper an investigation of the use of genetic algorithms into the problem of analog circuits design is presented. In a single design stage, circuits are produced that satisfy specific frequency response specifications using circuit structures that are unrestricted and with component values that are chosen from a set of preferred values. The extra degrees of freedom resulting from unbounded circuit structures create a huge search space. It is shown in this paper that Genetic Algorithms can be successfully used to search this space. The application chosen is a LC all pass ladder filter circuit design.
\end{abstract}

Key Words: Computer-Aided Design, Analog Circuits, Artificial Intelligence.

\section{INTRODUCTION}

Computer-aided design tools for analog circuits consist almost invariably of circuit analysis or simulation packages; no general-purpose analog circuit synthesis tools are available. Conventional numerical optimization techniques can be used to obtain combinations of component values that satisfy given design specifications. In addition, conventional optimization techniques operate on circuits after having their desired responses approximated by a suitable polynomial, using ideal components with unrestricted values, and only operating on pre-defined circuit structures. The restrictions introduced by pre-defining the circuit structure waste extra degrees of freedom in the design process and can conceal many novel design structures that can satisfy the target response and at the same time enhance the design process.

In previous papers, ${ }^{[1-6]}$ GA were applied to fixed structure circuit designs to satisfy certain specifications. As mentioned earlier, the predetermination of the circuit structure required in conventional design techniques is a limiting factor when considering a powerful search technique such as GA.

A few attempts have been made to include structure synthesis in genetically based optimization techniques such as Genetic Programming (GP) ${ }^{[7]}$ and GA ${ }^{[8]}$.
However, these techniques suffered huge delays and memory usage and needed to be used in conjunction with circuit analysis packages such as PSPICE ${ }^{[9]}$.

There is still a need, however, for more efficient algorithms that are faster, self-sufficient and can function independently. This is particularly important in the development of new generations of Analog Programmable Analog Arrays (APAA) Programming Tools. In this paper an efficient GA technique for free structure analog circuit design is described.

The paper is organized as follows. Section 2 introduces the Genetic Algorithms in details. Section 3 presents the GA implementation technique and chromosome structure, and section 4 discusses the Least Squared Error Evaluation technique used by GA. Section 5 presents results for a number of analog filter design specifications that were generated by the GA. Section 6 concludes the paper.

\section{GENETIC ALGORITHMS}

Genetic Algorithm is an evolutionary algorithm which falls into the class of global optimization techniques. A number of different classifications of the existing global optimization techniques are described in ${ }^{[10]}$. However, in ${ }^{[11]}$ a different classification is presented which would be more appropriate for the case considered here. It classified global optimization methods into two main categories, namely volume-oriented and path-oriented. In the volume-oriented method, an exhaustive search over the whole volume of the feasible region is conducted (e.g. grid search ${ }^{[12]}$, Monte-Carlo methods $\left.{ }^{[13]}\right)$. While in the path-oriented method, the search starts from an arbitrary or specifically chosen point in the feasible region, and then follows one or more paths in the search for a global optimum.

The path-oriented method is further divided into the prediction method and the Exploration method. The prediction method uses a model of the objective function to predict the steps (e.g. tunneling methods ${ }^{[14]}$ ), while the exploration method (e.g. rotating coordinates ${ }^{[15]}$ ) does not. Evolutionary Algorithms, according to the above classification, fall mainly into the group of path-oriented exploration methods. During the optimization process, however, evolutionary methods can act to a lesser degree as predicting methods as well as volume-oriented methods. The most common of these evolutionary algorithms is Genetic 
Algorithm which has gained much importance in the last twenty years ${ }^{[16]}$.

The field of Genetic Algorithms was founded by John Holland in the early 1970's. In [17], Holland emphasizes the ability of simple representations to encode complicated structures and the power of simple transformations to improve that structure. These representations are combined in what is called in biology a chromosome. A number of these chromosomes will constitute a population. Syntactic operations are then used to alter and improve these coded solutions.

Holland described Genetic Algorithms ${ }^{[18]}$ as a control structure with which these representations and operations could be managed in order to evolve bit strings that were well developed to the problem to be solved.

As in natural evolution, the problem each individual chromosome faces is one of searching for beneficial adaptations to a complicated and changing environment. The knowledge that each individual gains is embodied within the chromosomes of a population. Figure 1, shows a flowchart of a simple GA.

\section{IMPLEMENTATION}

\subsection{Chromosome Adaptation and Structure}

In [1-3] the predetermination of the circuit structures has helped in reducing the chromosome length. This was achieved by incorporating the order of the chromosome genes into the node connections information. However, in the case of free structure circuit synthesis this technique cannot be used.

In the case of free structure design, the solution space is much larger and the optimization process is composite. It involves the optimization of two different but highly correlated problems; the structure as well as the sizing of a circuit. Hence, all specifications of the electrical circuit including the structure and the sizing of all the components should be included in the chromosome representation.

The specifications of the electrical circuit include the number of components in the circuit, the type of each component and a list of connections between the components. Circuit components can include a variety of different types of components, including resistors, capacitors, inductors, etc..

Given the above information, the chromosome for each circuit comprises a number $\mathrm{M}$ of groups of equal bit lengths, and an extra group of bits representing the number of nodes in the circuit, Figure 2.

Each gene in the chromosome specifies a component and contains four fields (i) Type of component (L, C, etc.), (ii) A pointer to a menu containing component value, (iii) and (iv) circuit nodes to which the component is connected, see Figure 4.

It is generally desirable for a low cost design to use the least number of components. For this reason a Null component, representing an open circuit, is introduced into the component type menu.

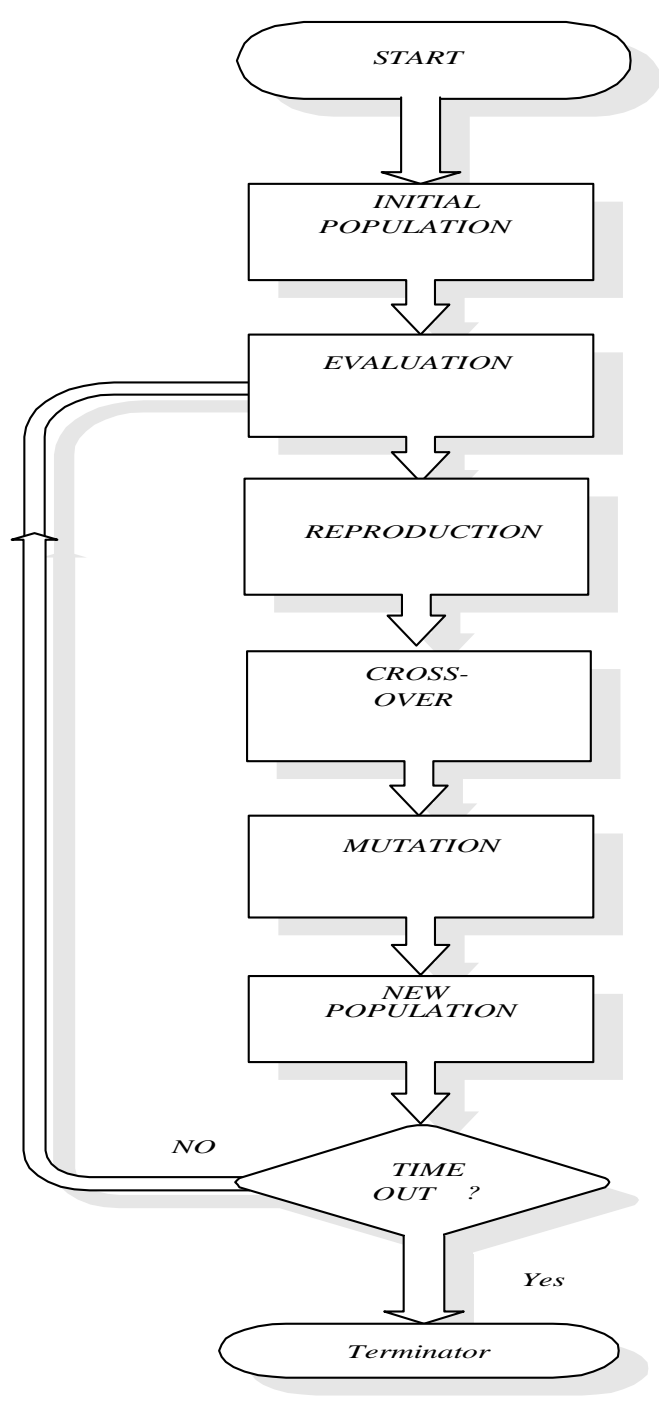

Fig. 1 Simple Genetic Algorithm

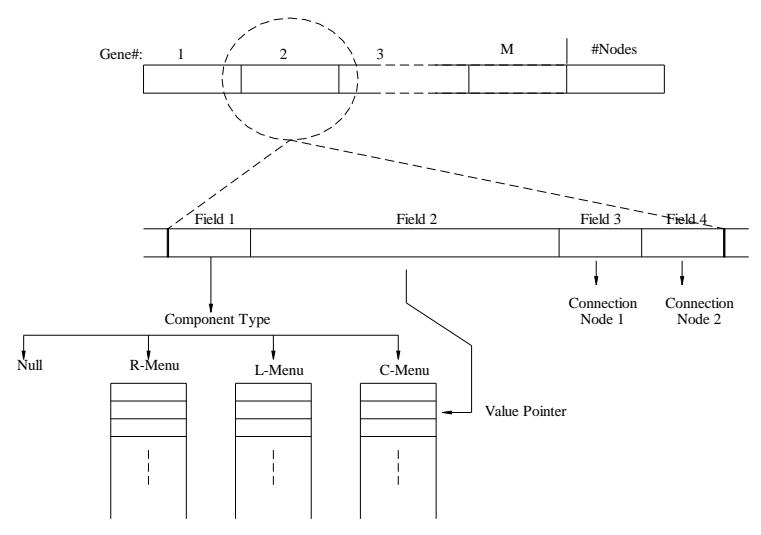

Fig. 2 Chromosome structure. 


\subsection{The Design Program}

The design program of the free structure circuit synthesis is briefly described in this section with pseudo code.

On entry the user is required to supply the following input data:

Type of filter (Low pass, High pass, Band pass)

$\omega_{1}, \alpha_{\min }$

$\omega_{2}, \alpha_{\max }$

- $\quad \mathrm{N}$, the maximum number of nodes. Optional, and can be used to limit the search to a certain number of designs.

- $\mathrm{M}$, the maximum number of components (optional). The program execution is described in pseudo code in Figure 3.

FOR population_member $=0$ to population_size, DO
Determine N, number of nodes
FOR gene_location $=1$ to $\mathrm{M}, \mathbf{D O}$
Decode fields $1,2,3$ and 4
Descale fields 3 and 4 according to N
Update NAM
END FOR
FOR frequency_test_point = 0 to maximum, DO
LU decompose NAM
Calculate response
Compare with template and find error
END FOR
END FOR

Fig. 3 Pseudo code for a single generation evaluation

The execution of the program starts by calculating N, maximum number of nodes. Then genes representing elements are decoded one at a time, splitting them into four fields as explained in section 5.1.

Upon determining type, value and connection nodes, the component value is inserted in the corresponding Nodal Admittance Matrix (NAM) location according to rules in section 4 . When all elements are decoded, contents of the resulting entries in NAM are passed to the calculation function, in which Lower-Upper (LU) decomposition and solutions to the matrix equations are solved.

The error and fitness evaluation functions are performed by means of least square error over a frequency design template specified as shown in the following section.

\section{LEAST SQUARED ERROR EVALUATION}

The error measured is defined as the difference between the specified response constraint, $H_{U p p}(\omega)$ and $H_{\text {Low }}(\omega)$, denoted by the shading in Figure 4, where $H_{U p p}(\omega)$ is the upper boundary of the frequency response, and $H_{\text {Low }}(\omega)$ is the lower boundary, and the actual response, $H_{a}(\omega)$, from the design represented by GA chromosomes. The actual frequency response, $H_{a}(\omega)$, will hence be prescribed by its unique combination of component values. Therefore,

$$
H\left(\omega, R_{1}, R_{2}, \ldots, R_{m}, C_{1}, C_{2}, \ldots, C_{n}, L_{1}, L_{2}, \ldots, L_{v}\right)
$$

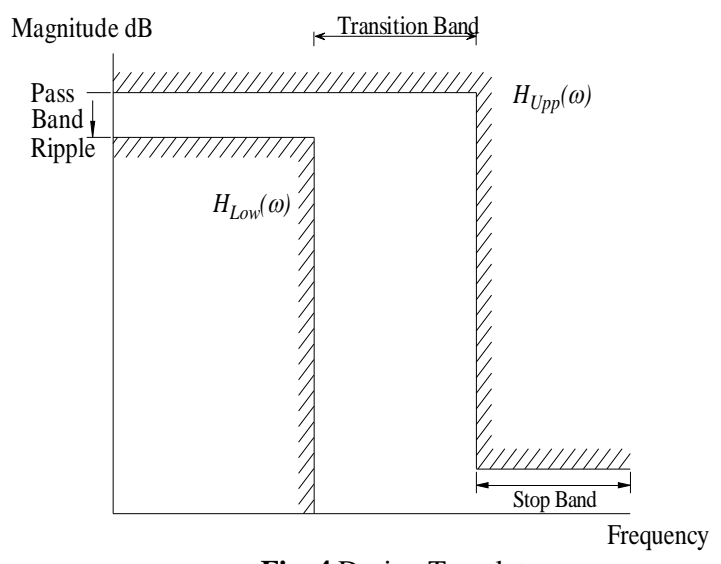

Fig. 4 Design Template

For simplicity, all types of components will be declared as k. Thus $H$ becomes

$$
H\left(\omega, k_{1}, k_{2}, \ldots, k_{q}\right)
$$

where $q=m+n+v$

Hence the error function will depend on the region it is applied for. For the pass band the error function would be

$$
\operatorname{err}\left(\omega, k_{1}, k_{2}, \ldots, k_{q}\right)=
$$

$$
H_{L o w}(\omega)-H_{a}\left(\omega, k_{1}, k_{2}, \ldots, k_{q}\right)
$$

while in the stop band the error function would be

$$
\begin{aligned}
& \operatorname{err}\left(\omega, k_{1}, k_{2}, \ldots, k_{q}\right)= \\
& H_{U p p}(\omega)-H_{a}\left(\omega, k_{1}, k_{2}, \ldots, k_{q}\right)
\end{aligned}
$$

The Least-Squares error criteria $\mathrm{E}$ is used as a performance index because of mathematical simplicity and ease of programming. The error is defined as

$$
E=\int_{l}^{u} \operatorname{err}\left(\omega, k_{1}, k_{2}, \ldots, k_{q}\right)
$$

Substituting from (13) in (12), LSE would be

$$
L S E=\int_{\omega_{l}}^{\omega_{u}}\left\{H(\omega)-H_{a}\left(\omega, k_{1}, k_{2}, \ldots, k_{q}\right)\right\}^{2}
$$

where $\mathrm{H}(\omega)$ is the boundary response $H_{U p p}(\omega)$ or $H_{\text {Low }}(\omega)$. Since the calculations must be performed 
numerically, the integration in Eq. (5) will be replaced by summation. Therefore,

$L S E=\sum_{i=1}^{m}\left[H(\omega)-H_{a}\left(\omega, k_{1}, k_{2}, \ldots, k_{q}\right)\right]^{2}$

where $m$ is equally spaced frequency points assumed over the range of integration. This is chosen because of ease in programming.

\subsection{Specified Weighted Errors}

Equation (6) gives equal weight for all frequency points within the range. This does not necessarily reflect the prescribed performance; for example, in the case of numerically small errors in the pass band. This numerically small error in the pass band will be more likely to affect the accuracy of a solution than a relatively larger error encountered in the stop band. Hence, a weight function can be inserted in equation (6) to enable us to emphasize or de-emphasize the error in the various regions of the frequency spectrum ${ }^{[19]}$. Therefore equation (6) can be modified to be

$$
L S E=\sum_{i=1}^{m}\left\{w(\omega) \cdot\left[H(\omega)-H_{a}\left(\omega, k_{1}, k_{2}, \ldots, k_{q}\right)\right]\right\}^{2}
$$

The weight function $w(\omega)$ has been arbitrarily chosen to be two in the pass band region and unity in the stop band region. Any other design weight functions can be easily applied instead.

Although the pass band will be characterized by a specific pass band ripple, the upper boundary of the response represents the maximum power transfer level, the claimed results of LC filter theory. Therefore, testing only the suitability of the lower limit of the response would be sufficient as the upper limit will never be exceeded in the pass band ${ }^{[11]}$.

In the stop band region, only the upper limit of the specified attenuation level needs to be considered, since any lower value of attenuation meets the specifications. The transition region is considered as a 'don't care' region. Thus it is sufficient to test just the pass band and the stop band for confirmations. The different frequency band boundaries are shown in Figure 4.

The fitness function is defined as the simple reciprocal of error as defined by Eq. (7). This produces the desired characteristic that fitness is larger for better circuits with less error, and also causes increased fitness separation for groups of solutions that are near optimum.

\subsection{Performance Improvements by Controlling Connection Fields}

The number of nodes comprising the circuit is determined at the beginning of the decoding process to control the connection node numbers of the circuit components.

The fields determining the connection nodes are decoded in a way to produce node numbers within $\mathrm{N}$. This is achieved by scaling down the whole range of values of allowed node numbers to only that of $\mathrm{N}$. This helps to avoid circuit structures that would have components connected to nodes that do not exist in the defined structure and which are hence unacceptable, and would be a total waste of time to evaluate.

\section{RESULTS}

It is very difficult and nearly impossible to design a practical circuit that meets the exact or the piecewise linear specifications of a given design. Therefore, $t$ is a common practice for circuit designers to specify a band of permissible variations in the gain characteristics rather than specific values for the gain at each frequency grid point.

In this implementation the desired design template is the starting point of the design process, whereas in conventional designs, polynomial approximation for the desired response is used ${ }^{[19]}$. This has the limitation of constraining the feasible solution to sets of design parameters that would satisfy the polynomial approximation. By designing directly from the template specifications a designer would avoid that extra degree of approximation a polynomial fitting would introduce. At the same time they provide an extra degree of freedom which would enlarge the solution space considerably to include any design that would reside within the design template without necessarily being represented by a polynomial. That would give Genetic Algorithms an advantage over conventional optimization methods for the credit it holds in investigating huge solution spaces, due to its nature. Also the GA search starts from an arbitrary point in the solution space and need not have any prior knowledge of the feasible region of the required design.

To illustrate the success of the above GA in designing filters with free topology, a number of design templates are specified upon which the GA are applied. A further design response is specified as a transfer function which the GA has successfully matched. In the cases of low and high pass design specifications, it is found that GA has provided solutions that have the conventional ladder structure circuits as well as some novel design structures, as will be seen in the next subsections. The flat losses of the circuit responses are left unconstrained. This is acceptable in many applications and the extra degree of freedom created tends to allow the GA to find more solutions.

\subsection{Low pass design}

Three different low pass design specifications are considered. In the first, a pass band ripple of $1 \mathrm{~dB}$, minimum attenuation of $-100 \mathrm{~dB}$ at a transition ratio of 10. An average group of five solutions emerged; the frequency response of one of these solutions is plotted in Figure 5. The circuit constructed by the GA is shown in Figure 6.

It is noticed that well known $\pi$ and $T$ ladder structures have also been generated by the GA. That could be due to the low sensitivity such structures have to small changes in component values. This feature will be discovered in GA as it approaches an acceptable solution. Large changes in the frequency response in a 
solution that could be considered as a good one will most likely affect its performance negatively, and hence the GA will tend to move away from that solution to a more stable solution region.

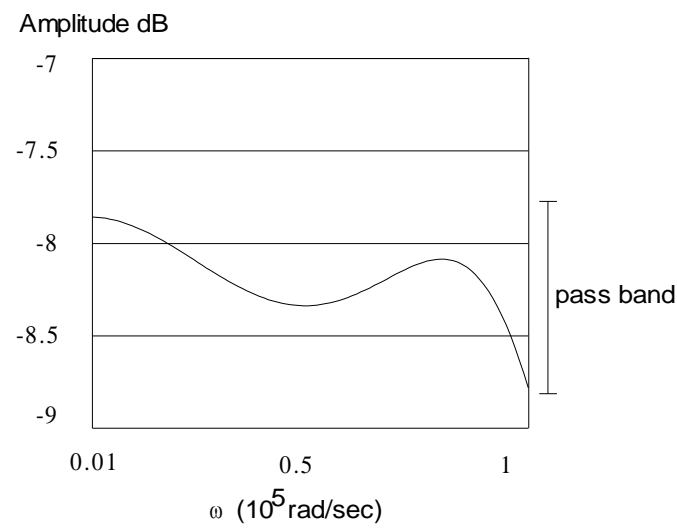

(a)

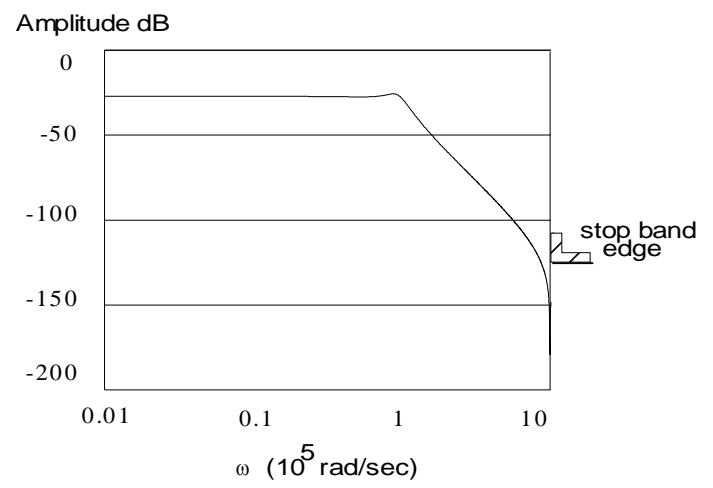

(b)

Fig. 5 Frequency response examples for low pass design template (a) pass-band and (b) stop band

A tighter specification in the pass band is introduced, with a $0.5 \mathrm{~dB}$ pass band ripple allowed and

$-60 \mathrm{~dB}$ minimum attenuation at pass band edge normalized at $10 \mathrm{rad} / \mathrm{sec}$. A group of five solutions also satisfied these specifications. A circuit structure of one of these is shown in Figure 7. The frequency response for three of the resulting families of circuits are shown in Figure 8 (a), (b), and (c).

A third template of $0.2 \mathrm{~dB}$ pass band ripple and stop band attenuation of $-50 \mathrm{~dB}$ with a transition ratio of 10 is also considered. A group of an average six solutions are generated. Some of these solution's frequency responses are plotted in Figure 9.

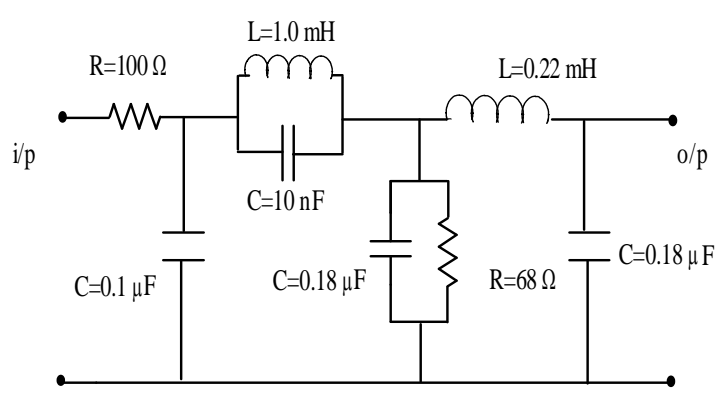

Fig. 6 Genetically designed circuit with a pass band ripple of $1 \mathrm{~dB}$ and stop band edge at $-100 \mathrm{~dB}$.

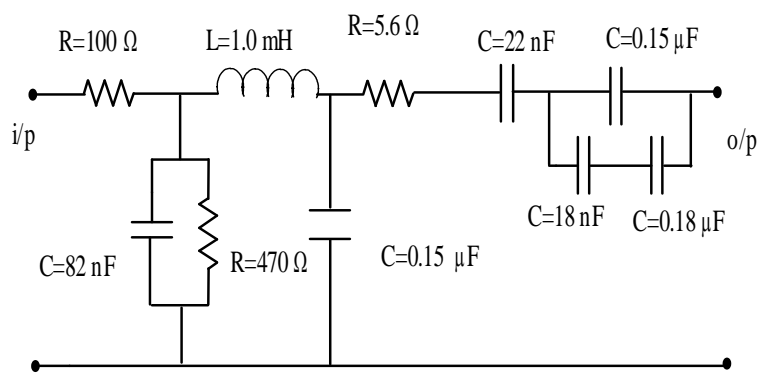

Fig. 7 Genetically designed filter circuit with $0.5 \mathrm{~dB}$ pass band ripple and $-60 \mathrm{~dB}$ stop band attenuation

\subsection{High pass design}

The high pass filter design is done directly without having to use a low pass prototype and then perform frequency transformations. A design template of $1 \mathrm{~dB}$ pass band ripple and attenuation of $-60 \mathrm{~dB}$ at the stop band edge with a transition ration of 10 is considered. Four solutions, on average, emerged. Two of these circuits are shown in Figure 10. The frequency responses of the resulting circuits are shown in Figure11.

\section{CONCLUSIONS}

This paper has presented a novel circuit design tool by using the GA strong search abilities in both the structure and sizing domain of a circuit design problem. A successful design of the chromosome structure has been obtained to include the full circuit specifications. 


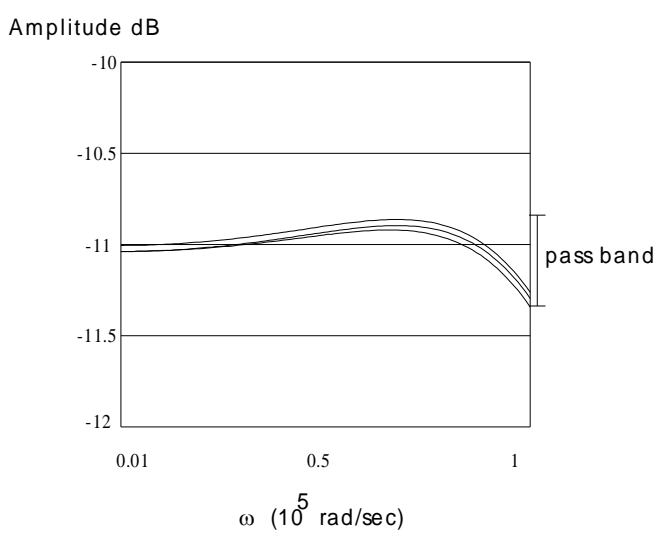

Amplitude dB

(a)

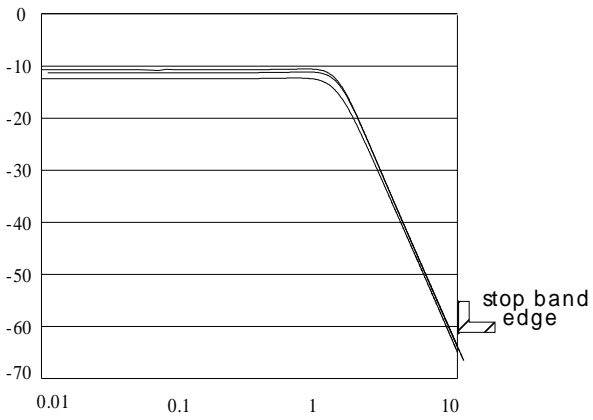

$\omega\left(10^{5} \mathrm{rad} / \mathrm{sec}\right)$

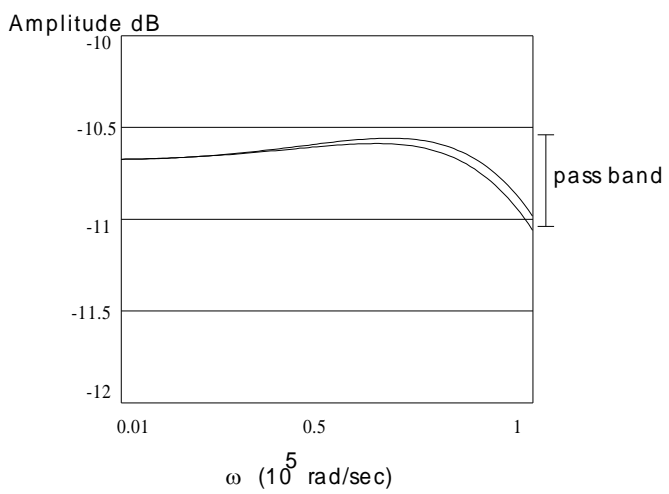

Amplitude dB

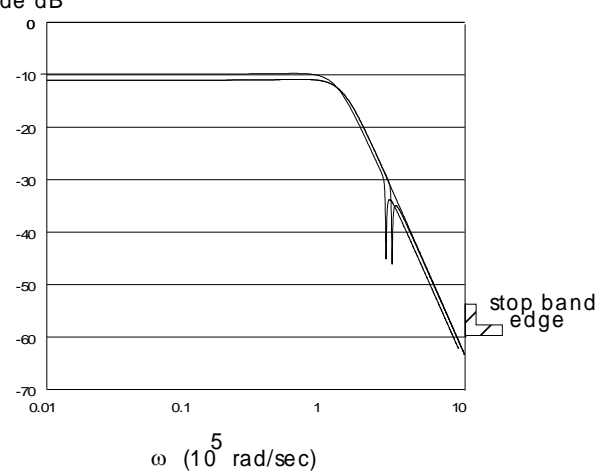

(b)

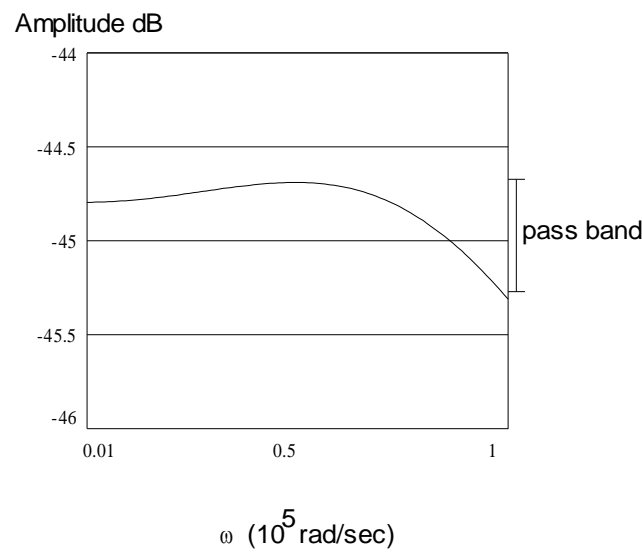

(c)

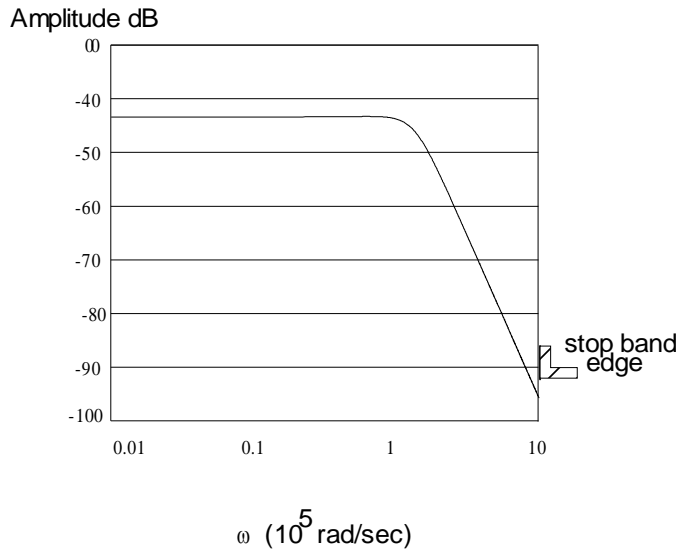

Fig. 8 (a), (b) and (c) frequency responses for low pass design template with $0.5 \mathrm{~dB}$ pass band ripple and $-60 \mathrm{~dB}$ stop band edge 

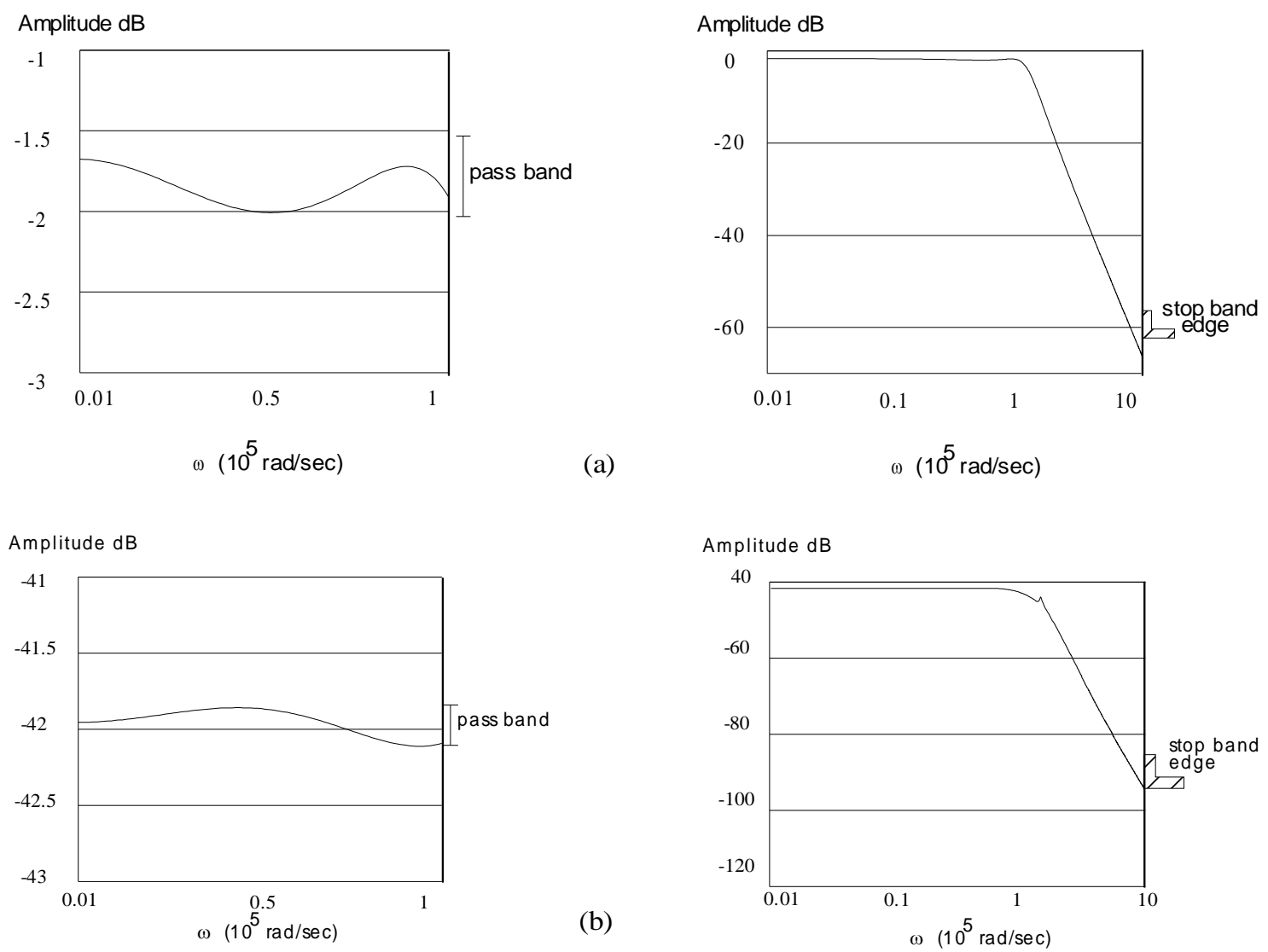

(b)

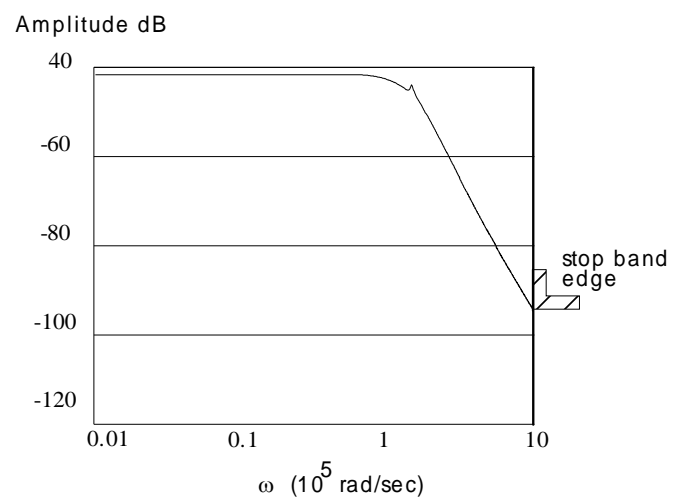

Fig. 9 (a) and (b) frequency response for low pass design template with a 0.2 band pass ripple and $-50 \mathrm{~dB}$ stop band edge

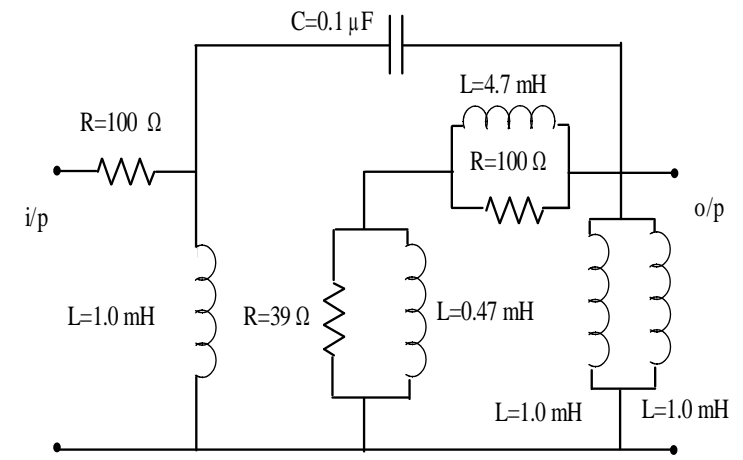

(a)

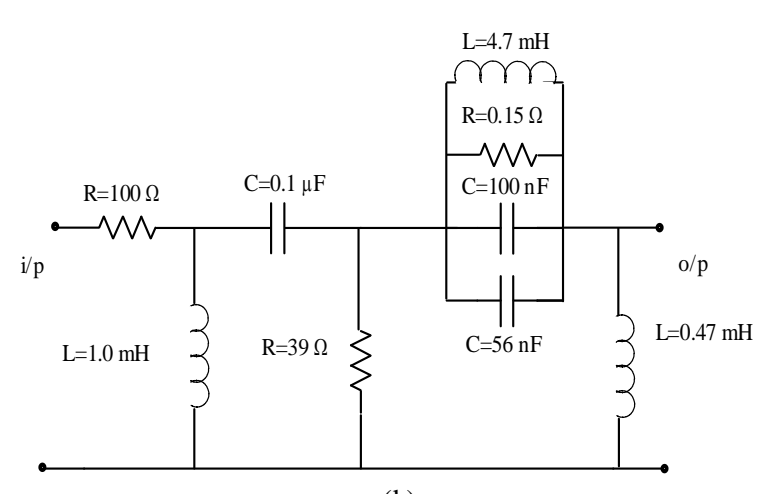

(b)

Fig. 10 (a) and (b) Genetically derived high pass filter circuit with $1 \mathrm{~dB}$ pass band ripple and attenuation of $-60 \mathrm{~dB}$ at the stop band edge 

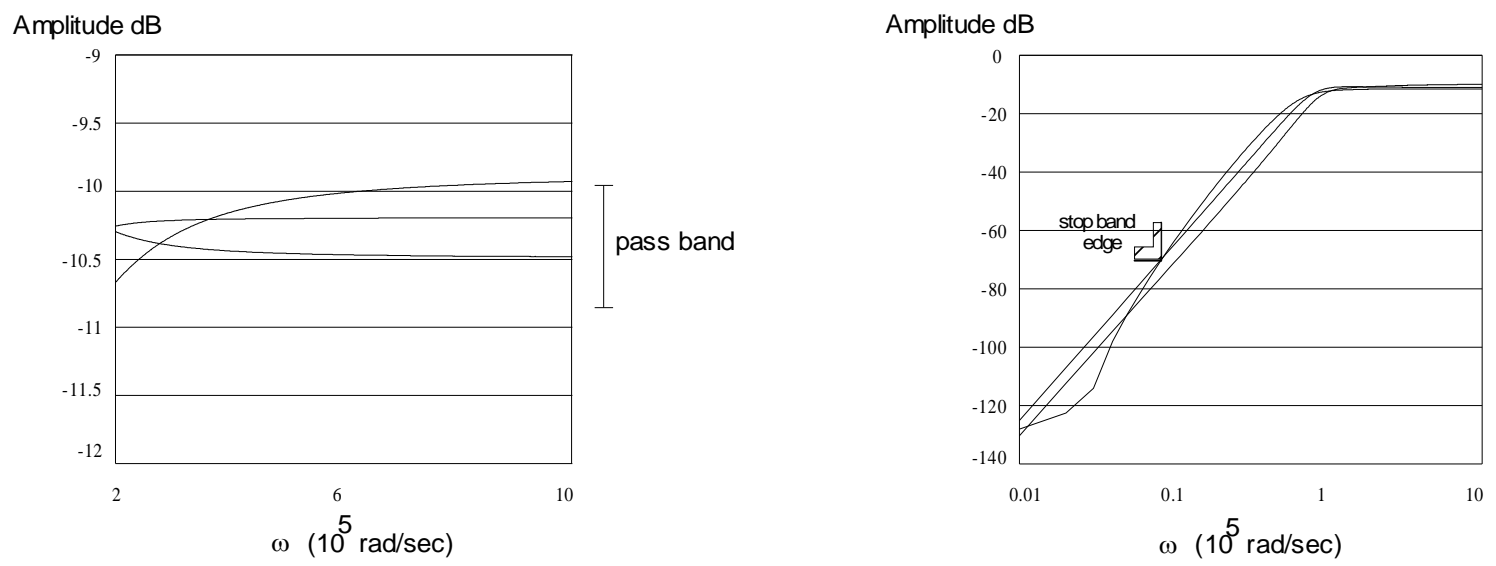

Fig. 11 Genetically derived high pass filter circuit

\section{REFERENCES}

[1] D. H. Horrocks and Y. M. A. Khalifa,, "Genetically Derived Filter Circuits using Preferred Value Components", Proc. IEE Colloquium on Analog Signal Processing, Oxford, UK, pp 4/1-4/5, Oct. 1994.

[2] D. H. Horrocks and Y. M. A. Khalifa," "Genetically Evolved FDNR and Leap-Frog Filters using Preferred Components Values", Proc. European Conference on Circuit Theory and Design, Istanbul, Turkey, pp 359362, Aug. 1995.

[3] D. H. Horrocks and Y. M. A. Khalifa,, "Genetic Algorithm Design of Electronic Analog Circuits Including Parasitic Effects", Proc. First On-line Workshop on Soft Computing (WSC1), Nagoya University, Japan, pp 71-78, Aug. 1996.

[4] D. H. Horrocks and Y. M. A. Khalifa,, "Evolutionary Design of Analog Electronic Circuits; Current Status", IEE Colloquium on Analog Signal Processing, Oxford, UK, pp 7/1-7/8, Nov. 1996.

[5] D. H. Horrocks and Y. M. A. Khalifa, "Rapid Feasible Region Estimation for Enhanced Performance of Genetically Derived Circuit Design Technique", Proc. of The Fourth IEEE International Conference on Electronics, Circuits and Systems, Cairo, Egypt, Dec. 1997.

[6] Y. M. A. Khalifa and D. H. Horrocks, "Isomorphism Elimination for the Enhancement of Genetically Generated Analog Circuits", Proc. of The International Symposium on Circuits and Systems ISCAS'99, Orlando, Florida. USA, pp 314-317, June 1999.

[7] J. R. Koza, D. Andre, F. H. Bennet III, and M. A. Keane,"Toward evolution of electronic animals using Genetic Programming", Proceeding of the first Annual Conference, Stanford University, Cambridge, July 2831, 1995.

[8] J. B. Grimbleby, "Automatic Synthesis of Active Electronic Networks using Algorithms", IEE Genetic Algorithms in Engineering Systems: Innovations and Applications, Conference Publication No. 446, PP103107, Sep. 1997.
[9] W. Blume, "Computer Circuit Simulation", Byte v $11 \mathrm{n}$ 7, pp. 165-166, 168-170, Jul 1986.

[10] G. S. G. Beveridge and G. Schechter, Optimization theory and practice, New York, McGraw-Hill, 1970.

[11] K. Ensor, G. Bennet and W. Peter, "Stochastic optimization via grid search", Proc. Mathematics of Stochastic Manufacturing Systems, Williamsburg, VA, pp. 89-100, 1996.

[12] J. P. C. Kleijnen, Statistical techniques in simulation, M. Dekker, New York, 1974-75.

[13] A. Levy and V. S. Gomez, "The tunnelling method applied to global optimization", Numerical Optimization, pp. 213-244, 1984.

[14] A. M. Arthurs and J. T. Lewis, "Rotating co-ordinates in scattering theory", Proceedings of the Royal Society. Series A. Mathematical and Physical, Sciences A 269, pp 585-588, , 1962.

[15] D. E. Goldberg, Genetic Algorithms in Search, Optimization and Machine Learning, Addison-Wesley, Reading MA USA, 1989.

[16] J. Holland, Adaptation in natural and artificial systems, University of Michigan, Ann Harbor, 1975.

[17] L. Davis, Handbook of Genetic Algorithms, Van Nostrand Reinhold, New York, pp. 36-38, 1991.

[18] L. O. Chua, Computer-Aided Analysis of Electronic Circuits, Prentice-hall, 1975.

[19] G. C. Times and J. W. LaPatra, Circuit Synthesis and Design, McGraw-Hill, New York, 1977.

\section{APPENDIX}

\section{Direct Construction of Nodal Admittance Matrix and Current Source Vector}

In the actual implementation of nodal analysis on digital computers, a direct method which avoids the complexity of constructing $\mathrm{A}$ and $\mathrm{Y}_{\mathrm{b}}$ and performing the matrix multiplication is used ${ }^{[19]}$. 
The rules for forming the nodal equations by inspection can be stated as follows:

1. The diagonal entries of $\mathrm{Y}$ are positive and

$$
\mathrm{y}_{\mathrm{jj}}=\sum \text { Admittances connected to node } \mathrm{j}
$$

2. The off diagonal entries of $\mathrm{Y}$ are negative and are given by

$\mathrm{y}_{\mathrm{jk}}=-\sum$ Admittances connected between node $\mathrm{j}$ and $\mathrm{k}$

3. The $\mathrm{j}^{\text {th }}$ entry of the right-hand-side vector $\mathbf{J}$ is: $\mathbf{J}_{\mathrm{j}}=\sum$ Current from independent sources entering node $\mathrm{j}$

The above are useful when formulating network equations by hand as they enable us to write the equations on a node-by-node basis. For computer formulation, an element-by-element approach is preferable, as the equations are set up in a single scan of the element list. Consider an element with admittance $\mathrm{Y}$ connected between nodes $\mathrm{j}$ and $\mathrm{k}$.

Let the current through this element be denoted by $i$ with orientation from node $\mathrm{j}$ to node $\mathrm{k}$. The current $i$ will appear only in the KCL equations associated with nodes $\mathrm{j}$ and $\mathrm{k}$, once with a positive sign and once with a negative sign

KCL at node $\mathrm{j}: \ldots+i+\ldots=\ldots$

$\mathrm{KCL}$ at node $\mathrm{k}: \ldots-i+\ldots=\ldots$

Let us now write the current $i$ in terms of the voltage across the element, $v_{j}^{-} v_{k}$, and the admittance.

KCL at node $\mathrm{j}: \ldots+y\left(v_{j}-v_{k}\right)+\ldots=\ldots$

KCL at node $\mathrm{k}: \ldots-y\left(v_{j}-v_{k}\right)+\ldots=\ldots$

Separating the term associated with the voltages,

$\mathrm{KCL}$ at node $\mathrm{j}: \ldots+y v_{j}+\ldots-y v_{k} \ldots=\ldots$

KCL at node $\mathrm{k}:{ }^{\ldots-y v_{j}+\ldots+y v_{k} \ldots=\ldots}$

We thus find that admittance of a two terminal element connected between nodes $\mathrm{j}$ and $\mathrm{k}$ appears only in rows and columns $\mathbf{J}$ and $\mathrm{k}$ of $\mathrm{Y}$ with a plus sign at locations $(\mathrm{j}, \mathrm{j})$ and $(\mathrm{k}, \mathrm{k})$ and with a negative sign at locations $(j, k)$ and $(k, j)$. Symbolically, this is written as

$$
\begin{aligned}
& j \\
& j \\
& k \\
& \left.k \begin{array}{cc}
y & -y \\
-y & y
\end{array}\right]
\end{aligned}
$$

For the right-hand-side vector we note that an independent current source of value $\mathrm{J}$ connected between nodes $\mathrm{j}$ and $\mathrm{k}$ (oriented toward $\mathrm{k}$ ) contributes to rows $\mathrm{j}$ and $\mathrm{k}$ of $\mathbf{J}$ as follows
$k\left[\begin{array}{c}-J \\ J\end{array}\right]$

The symbolic representation (7) and (8) are the key to computerized formulation and will be extended to

\begin{tabular}{|c|c|}
\hline$\alpha_{\max }$ & Maximum attenuation in the pass-band \\
\hline$\alpha_{\min }$ & Minimum attenuation in the stop-band \\
\hline$\omega_{1}$ & Pass-band angular frequency edge \\
\hline$\omega_{2}$ & Stop-band angular frequency edge \\
\hline$A$ & Incident matrix \\
\hline$E$ & Total error function \\
\hline $\operatorname{err}(\omega)$ & Error function at angular frequency $\omega$ \\
\hline$H_{u p p}(\omega)$ & $\begin{array}{l}\text { Design template upper bound for the } \\
\text { frequency response }\end{array}$ \\
\hline$H_{\text {low }}(\omega)$ & $\begin{array}{l}\text { Design template upper bound for the } \\
\text { frequency response }\end{array}$ \\
\hline$H_{a}(\omega)$ & $\begin{array}{l}\text { Actual frequency response of the resulting } \\
\text { circuit }\end{array}$ \\
\hline$i$ & Element current \\
\hline$J_{n}$ & Equivalent nodal current source vector \\
\hline$K C L$ & Kirchof Current Law \\
\hline$L U$ & Lower-Upper matrix decomposition \\
\hline$M$ & $\begin{array}{l}\text { Maximum Number of components in a } \\
\text { chromosome }\end{array}$ \\
\hline$N$ & $\begin{array}{l}\text { Maximum number of nodes in a } \\
\text { chromosome }\end{array}$ \\
\hline$N A M$ & Nodal Admittance Matrix method \\
\hline$q$ & Total number of components in a circuit \\
\hline$v$ & Element voltage \\
\hline$Y_{b}$ & Branch admittance \\
\hline$Y_{n}$ & Nodal admittance matrix \\
\hline$y_{j j}$ & Admittance connected to node $\mathrm{j}$ \\
\hline$y_{j k}$ & Admittance connected between 1 \\
\hline
\end{tabular}
other kinds of elements.

\section{LIST OF SYMBOLS AND ABBREVIATIONS}

\section{BIOGRAPHY}

Yaser M. A. Khalifa received his B. Sc. degree from Alexandria University, Egypt, and his $\mathrm{PhD}$ degree from Cardiff University of Wales in 1992 and 1997 respectively. Dr. Khalifa is currently an Assistant Professor at the department of Electrical Engineering at the University of North Dakota, USA. Dr. Khalifa's primary research interest is in the application of evolutionary algorithms in the design of analog and digital circuits.

David H. Horrocks obtained a B.Sc. (hons) from the University of Westminster, London, in 1962 and his $\mathrm{Ph}$.D. from the University of Newcastle upon Tyne, UK, in 1968 and then held a postdoctoral research post. Since 1970, he has been on the faculty of Cardiff University until his recent retirement. His research interests include analog and digital signal processing; AI techniques for electronic design, and low power signal processing. He is an author/co-author of two books, several patents and 100 research publications. He was co-recipient of the 1990/91 IEE Ambrose Fleming Premium journal paper award. 